\title{
A MEASUREMENT METHOD FOR DETERMINING THE DIRECTION OF PROPAGATION OF FLICKER AND FOR TRACING A FLICKER SOURCE
}

\author{
Peter G.V. Axelberg \\ Unipower AB \\ Alingsås, Sweden \\ peter.axelberg@unipower.se
}

\author{
Math H.J. Bollen \\ Luleå University of Technology \\ Skellefteå, Sweden \\ m.bollen@ieee.org
}

\author{
Irene Y.H. Gu \\ Chalmers University of Technology, \\ Gothenburg, Sweden \\ irenegu@s2.chalmers.se
}

\begin{abstract}
INTRODUCTION
This paper describes a new and highly efficient measurement method (algorithm) that determines how flicker propagates throughout the network and also traces the dominant flicker source. The fundamental principle of the method is to use the fact that a flicker source produces an amplitude modulation in the voltage and current waveforms. The low frequency variations in voltage and current that cause flicker are retrieved in a demodulation and filtering process. By first multiplying the low frequency variations in voltage and current and then integrate, a new quantity, flicker power, is achieved. The sign and the magnitude of flicker power give the direction to the flicker source as well as tracing the dominating flicker source.
\end{abstract}

\section{BACKGROUND}

Flicker is a power quality phenomenon that can affect our daily lives. A small and repetitive change in the intensity of a light source can be very annoying for a human being particularly when reading. Light-intensity fluctuations are due to voltage fluctuations which in turn are due to fluctuating power demands. A load causing flicker is often connected to the power network at medium- or high voltage level resulting in flicker propagation throughout a wide area of the network affecting a large number of customers. Typical examples of such loads are arc furnaces, welding machines but also wind turbines [1-6]. It is of mutual interest for both the network operator and the customers to have a complete picture of the dominating flicker sources. Any disagreement can slow down a mitigation process. In many cases it is quite obvious which load is the dominating flicker source but in other cases it is not. For example steel plants are in many places (like in Sweden) located quite close to each other and connected to the same grid. That means the actual flicker level measured at the point of common coupling (PCC) is a result of power fluctuations originating from all possible flicker sources (in this case the steel plants). The contribution from each particular steel plant depends mainly on the magnitude and frequency of the fluctuations and the power network structure (strong or weak network). The dominating flicker source (i.e. the steel plant that contributes most to the flicker level) will also change over time depending on the operational stage of the steel plant and also on the stage in the melting process. The most common way to measure the flicker level is by using a flickermeter defined in the standard IEC 61000-4-15 [7-8]. The input to the flickermeter is the voltage waveform at the monitoring point and the output are the flicker values $\mathrm{P}_{\mathrm{st}}$ and $\mathrm{P}_{\mathrm{lt}}$. Since the input signal to the flickermeter is only the voltage waveform, it is not possible to determine how flicker propagates in the power network or to determine the dominating flicker source. A measurement method for determining the propagation of flicker and tracing a flicker source is therefore highly demanded. The method proposed in this paper uses the same signal processing chain as in IEC 61000-4-15 but for both voltage and current waveforms. The output signals from the signal processing chains are the low frequency variations in voltage and current that causes flicker. By first multiplying and then integrating the output signals a new quantity, flicker power, is obtained. This new quantity has the same attributes as active power. That means it is possible to determine the flicker power flow direction (i.e. propagation) as well as to trace a dominating flicker source. Only a few works has been presented within this topic before [9-11] but all of them have a different approach compared to the method proposed in this paper.

\section{THE PROPOSED METHOD}

The proposed method is derived from the circuit model shown in Figure 1.

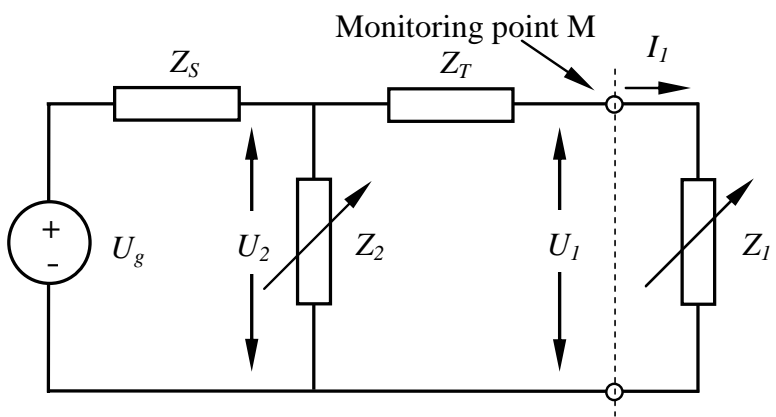

Figure 1. Circuit model used to derive the proposed method.

The model is basically a generator - load chain consisting of an independent voltage source $\left(U_{g}\right)$ with the internal impedance $Z_{S}$, a transmission line $Z_{T}$ and the two loads $Z_{l}$ and $Z_{2}$. Close to $Z_{l}$ is the monitoring point (M) where the voltage $U_{l}$ and current $I_{l}$ are monitored. The left side of $\mathrm{M}$ is referred to as "above monitoring point" and the right side to as "below 
monitoring point". The circuit model is considered to be a linear network which means that basic circuit theory laws like Ohm's law and Kirchoff's laws can be used when deriving the method. The two load sources $Z_{1}$ and $Z_{2}$ are understood as a combination of flicker sources with fluctuating power demand and loads with constant power demand. That means when the flicker sources are in a non-operating state, power is still demanded to feed the loads with constant power demand. A fluctuating power demand either from $Z_{1}$ or from $Z_{2}$ will immediately result in a fluctuation in the voltage $U_{l}$ and current $I_{l}$ at monitoring point $\mathrm{M}$. Two cases will now appear depending on if the flicker source is placed below $\left(Z_{l}\right)$ or above $\left(Z_{2}\right)$ the monitoring point $\mathrm{M}$.

Case 1: The flicker source is placed below monitoring point M (also referred to as "downstream"). Using Ohm's law and Kickoff's laws to derive $U_{l}$ and $I_{l}$ yields that when the load current $I_{l}$ increases the voltage $U_{l}$ will decrease due to increased voltage drop across $Z_{S}$ and $Z_{T}$

$$
I_{1}+\Delta I_{1} \rightarrow U_{1}-\Delta U_{1}
$$

Case 2: The flicker source is placed above monitoring point $\mathrm{M}$ (also referred to as "upstream"). The voltage $U_{2}$ is changing when $\mathrm{Z}_{2}$ changes. Since $U_{2}$ supplies the circuit branch consisting of $Z_{T}$ and $Z_{1}$ a change in $U_{2}$ will immediately result in a change of both $I_{l}$ and $U_{l}$ according to:

$$
U_{2} \pm \Delta U_{2} \quad \rightarrow \quad I_{1} \pm \Delta I_{1} \quad \rightarrow \quad U_{1} \pm \Delta U_{1}
$$

Two important observations can be stated from the results in (1) and (2). Firstly, the voltage and current signals are amplitude modulated due to the low frequency fluctuations $\Delta U$ and $\Delta I$. Secondly, the low frequency fluctuations are either $180^{\circ}$ out-of-phase (case 1) or in-phase (case 2) depending on if the flicker source is placed below or above the monitoring point $\mathrm{M}$. These two observations are the cornerstones in the proposed method. The amplitude modulated signals in voltage and current are expressed as:

$$
\begin{gathered}
u_{A M}(t)=\left(U_{c}+m_{u}(t)\right) \cdot \cos \left(\omega_{c} t\right) \\
i_{A M}(t)=\left(I_{c}+m_{i}(t)\right) \cdot \cos \left(\omega_{c} t\right)
\end{gathered}
$$

The low frequency fluctuations are represented as $m_{u}(t)$ and $m_{i}(t)$ in (3) and (4). According to the flicker meter standard IEC 61000-4-15 the human sensitivity to flicker is a function of both modulating frequency and degree of modulation. That means the low frequency signals $m_{u}(t)$ and $m_{i}(t)$ must be filtered according to how the human responds to flicker. This is achieved by using the sensitivity filter described in the IEC 61000-4-15. The output signals $m_{u \Pi}(t)$ and $m_{i \Pi}(t)$ indicate how an average human responds to flicker. By multiplying and integrating $m_{u I}(t)$ and $m_{i \Pi(}(t)$ a new quantity flicker power or $\Pi$ is achieved:

$$
\Pi=\frac{1}{T} \int_{0}^{T} m_{u \Pi}(t) \cdot m_{i \Pi}(t) d t
$$

This new quantity has the same attributes as active power. For example flicker power has a flow direction. With respect to a monitoring point $\mathrm{M}$, positive sign of flicker power means a flow direction from generator towards the load whilst negative flicker power sign means a flow direction in the opposite direction (i.e. from load towards the generator). Furthermore flicker power is a conserved quantity if demodulation is performed in a correct manner. That means, the algebraic sum of the flicker powers entering and leaving any node is zero. The first attribute is used to trace the dominating flicker source and the second attribute makes it possible to compare flicker power from different monitoring points (feeders) and immediately determine the feeders that contribute most to the actual flicker level.

\section{Signal flow diagram of the proposed method}

The proposed method that has been implemented and tested in practice is based on the above discussion. The signal flow diagram is shown in Figure 2.

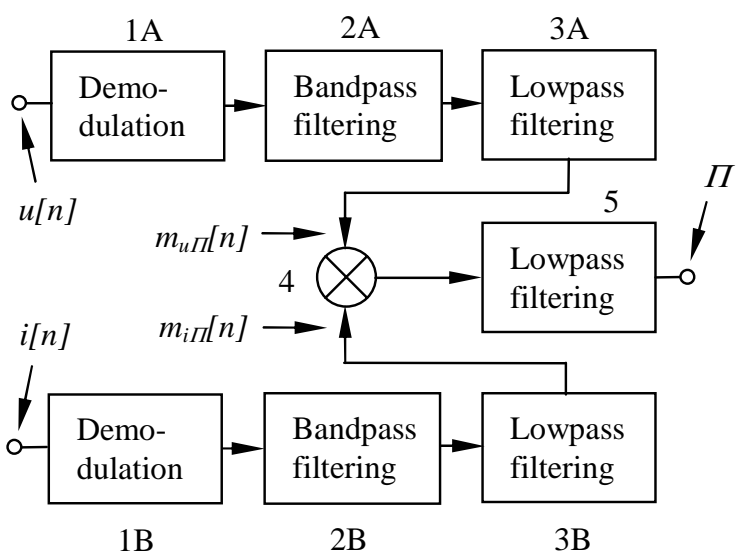

Figure 2. Signal flow diagram of the proposed method.

The input signals $u[n]$ and $i[n]$ are sampled versions of the analogue measured signals and applied to two identical chains (block 1 to 3 ). Block 1 is a demodulator creating a new frequency spectrum where the low frequency signals are retrieved and (well) separated from the $50 \mathrm{~Hz}$ carrier signal. Next, the demodulated signals are applied to two digital filters in cascade (block 2 and 3). The first filter is the flicker sensitivity filter which transfer function is defined in IEC 61000-4-15. The second filter is a lowpass filter used for further attenuation of the signal components outside the flicker frequency window. The output signals $\left(m_{u \Pi[}[n]\right.$ and $m_{i \Pi}[n]$ in Figure 2) from each filter chain is first multiplied (block 4) and then averaged by a low pass filter (block 5) in order to finally obtain the flicker power $\Pi$.

\section{VALIDATION OF THE PROPOSED METHOD}

The proposed method has been validated both by simulations 
in MATLAB and in several field tests all with very promising results. The field test presented in this paper was performed in a 130kV substation located in Sandviken, Sweden (Figure 3). The substation consists of two incoming lines from Stackbo and Forsbacka and five outgoing lines. The flicker power was measured on phase A on all seven lines simultaneously at the monitoring points M1 to M7 in Figure 3. Two of the outgoing lines (at M5 and M6) are feeding a 75 MW arc furnace via step-down transformers. Another outgoing line (at M7) is feeding the village of Hofors and industrial facilities approximately $25 \mathrm{~km}$ away from the substation. One major customer on that line is another arc-furnace situated in Hofors. The remaining two outgoing lines (at M3 and M4) are feeding major industrial facilities in the area close to the substation via step-down transformers.

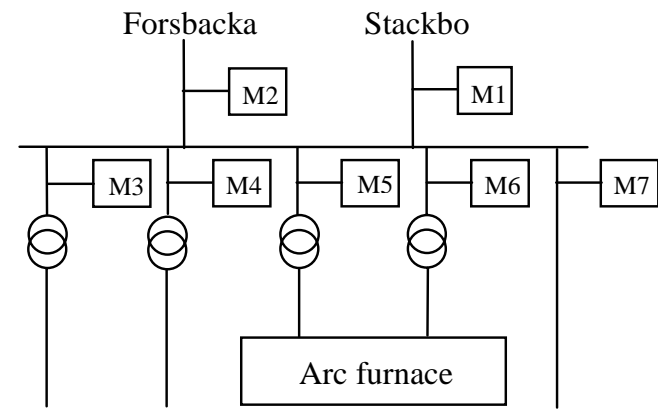

Industrial facilities

Hofors

Figure 3. One-line diagram of the $130 \mathrm{kV}$ substation in Sandviken,
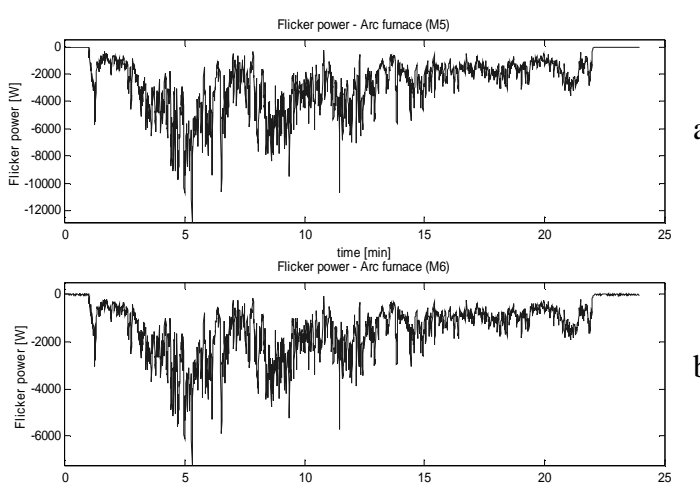

Flicker power- Forsbacka (M1)
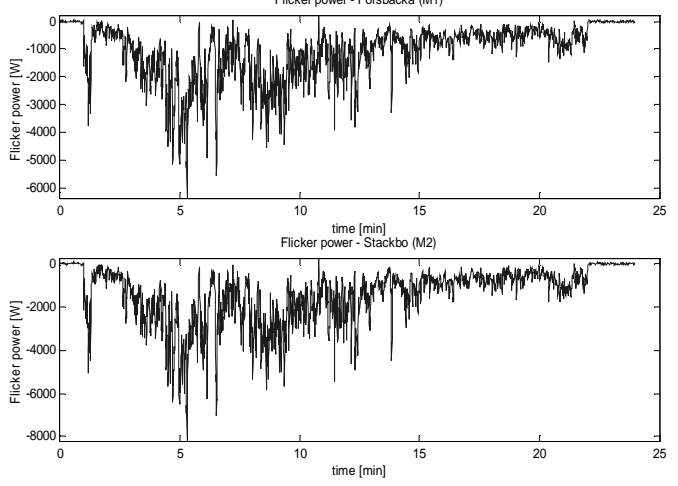

Figure 4. Measurement results from monitoring points producing negative flicker power. Diagram a) and b) show the flicker power measured at the lines feeding the arc furnace and diagram c) and d) show the flicker power flowing in the incoming lines.
The measurement was conducted during one melting cycle of the arc furnace so there was no doubt which load was the dominating flicker source. Negative flicker power was therefore expected at the two lines feeding the arc furnace since the flicker source (i.e. arc furnace) is located below the monitoring points M5 and M6. Negative flicker power is also expected at the two incoming lines since the arc furnace is placed below the monitoring points M1 and M2. The flicker power measured from the remaining lines is expected to be positive since the flicker source is placed above the monitoring points M3, M4 and M7. The measurement results are shown in Figure 4 and Figure 5 and they are very much the expected ones. As shown in Figure 4 the correlation between produced flicker power from the arc-furnace and the flicker power propagated to the incoming lines is significant. The results in Figure 5 are also the expected ones since positive flicker power is measured at the monitoring points M3 and M4 which means the flicker power flow direction is from the bus into these two lines. Even more interesting is the flicker power measured at the outgoing line to Hofors. Figure $5 \mathrm{c}$ shows a quite fluctuating flicker power between 4 and 11 minutes with partly negative flicker power during that time. In order to explain this some more information must be included in the analysis: As mentioned earlier the outgoing line to Hofors is also feeding an arc-furnace situated in Hofors. That means the flicker power measured at monitoring point M7 will be a result of interaction from two arc-furnaces. The conclusion is that the arc furnace in Hofors is partly the dominant flicker source at that line. That means the flicker power measured at monitoring point M7 will be a result of interaction from two arc-furnaces.
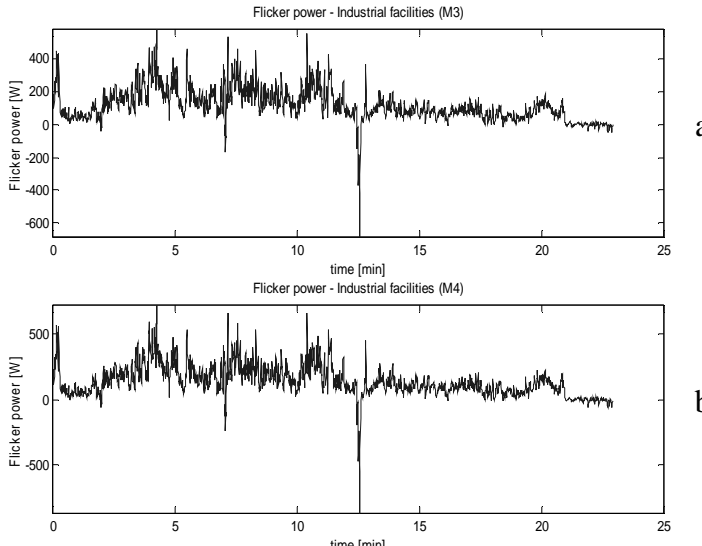
time $[$ min]
Flicker power - Hotors (M7)

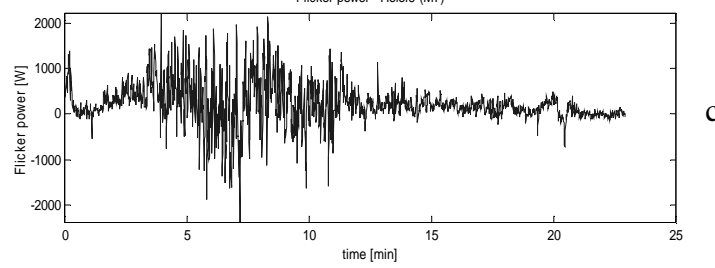

Figure 5. Measurement results from monitoring points producing mainly positive flicker power. The flicker power in diagram c) (i.e. the outgoing line to Hofors) is fluctuating heavily when the produced flicker power at the arc furnace in Sandvik is low and fluctuating. The reason is that the arc-furnace in Hofors is operating at the same time and contributes to the total flicker power level at monitoring point M7 (see current RMS plot in Figure 6). 
Looking at the RMS current of the outgoing line to Hofors (Figure 6) it is most certain that the arc furnace in Hofors starts to operate after 6 minutes due to the heavy fluctuations in the RMS current. At the same time the arc furnace at Sandvik is producing only a small amount of flicker power and the resulting flicker power at monitoring M7 is sometimes negative (i.e. the arc furnace in Hofors is the dominant flicker source at that time). When the RMS current stabilizes, the flicker power measured at M7 also stabilizes.

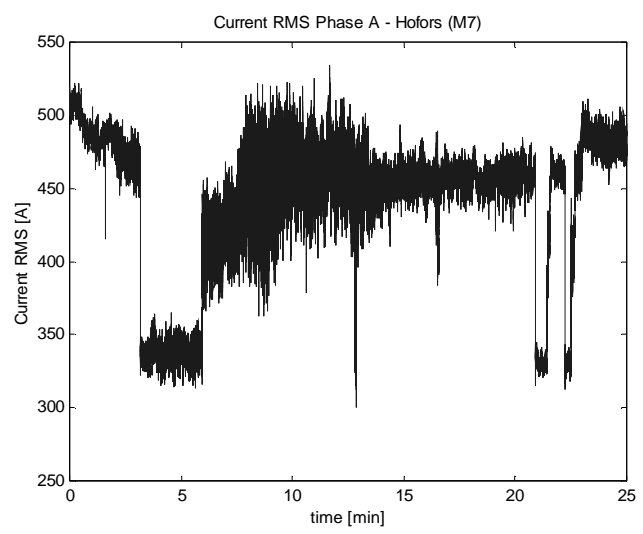

Figure 6. Diagram of the RMS current of the outgoing line to Hofors (M7). The heavy fluctuation that starts after 6 minutes is certainly due to the operation of the arc furnace located in Hofors. The fluctuations in current will result in flicker power fluctuations shown in Figure $5 \mathrm{c}$.

\section{SUMMARY AND CONCLUSIONS}

A new and highly efficient method has been developed for determining the direction of propagation of flicker and for tracing a flicker source. The proposed method is basically a development of the flicker meter standard IEC 61000-4-15. Demodulation and filtering is performed in the same way but with the important extension that the current waveform is also included in the signal processing chain. The low frequency variations in voltage and current are first filtered and then multiplied and averaged to get the new quantity: flicker power. This new quantity is shown to have the same attributes as active power. The sign tells the flow direction of flicker power with respect to a monitoring point and the magnitude makes it possible to compare flicker power from different monitoring points and thereby gives an important contribution to trace the dominating flicker source. Both simulations and field tests have been performed and the results were fully in line with the developed theory.

\section{REFERENCES}

[1] P. Axelberg, 2003, Measurement Methods for Calculation of the Direction to a Flicker Source. Thesis for the degree of Licentiate of Engineering, Chalmers University of Technology, Dept. of Electric Power Engineering, Gothenburg, Sweden.
[2] P. Ashmole, 2000, Quality of supply - voltage fluctuations. Power Engineering Journal, June 2000, p 113-119.

[3] A. Larsson, 2002, Flicker Emission on Wind Turbines During Continuous Operation. IEEE transactions on Energy Conversion, Vol 17, No 1, 2002, p 114-118.

[4] T. Thiringer, 2004, Flicker Contributions from Wind Turbine Installations. IEEE transactions on Energy Conversion, Vol 19, No 1, 2002, p 157-163.

[5] S. Mendis, 1996, Investigations of Voltage Flicker in Electric Arc Furnace Power Systems. IEEE Industry Applications Magazine, January/February 1996, p 2834.

[6] J. Schlabbach, D. Blume, T. Stephanblome, 2001, Voltage quality in electric power systems. London: Institution of Electrical Engineers.

[7] IEC, 1998, Standard IEC 61000-4-15:1998, Flickermeter - Functional and design specifications.

[8] IEC, 2003, IEC 61000-4-15/A1, amendment No 1 to standard IEC 61000-4-15:1998.

[9] A.M. Dán, 1998, Identification of a Flicker Source, Int. Conf. on Harmonics and Quality of Power, ICHQP '98, Athens, Greece, 1998.

[10] B. Hughes, 2000, Source identification of voltage sag and flicker. IEEE Power Engineering Society, Summer Meeting, 2000.

[11] K. Srinivasan, 1995, RMS Fluctuations Attributable to a single customer. Power Quality Solutions, September 1995. 